\title{
Nanoscale
}

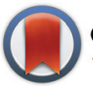

CrossMark \&lick for updates

Cite this: Nanoscale, 2015, 7, 14953

\section{Quantification and modification of the equilibrium dynamics and mechanics of a viral capsid lattice self-assembled as a protein nanocoating $\dagger$}

\begin{abstract}
Alejandro Valbuena and Mauricio G. Mateu*
Self-assembling, protein-based bidimensional lattices are being developed as functionalizable, highly ordered biocoatings for multiple applications in nanotechnology and nanomedicine. Unfortunately, protein assemblies are soft materials that may be too sensitive to mechanical disruption, and their intrinsic conformational dynamism may also influence their applicability. Thus, it may be critically important to characterize, understand and manipulate the mechanical features and dynamic behavior of protein assemblies in order to improve their suitability as nanomaterials. In this study, the capsid protein of the human immunodeficiency virus was induced to self-assemble as a continuous, single layered, ordered nanocoating onto an inorganic substrate. Atomic force microscopy (AFM) was used to quantify the mechanical behavior and the equilibrium dynamics ("breathing") of this virus-based, self-assembled protein lattice in close to physiological conditions. The results uniquely provided: (i) evidence that AFM can be used to directly visualize in real time and quantify slow breathing motions leading to dynamic disorder in protein nanocoatings and viral capsid lattices; (ii) characterization of the dynamics and mechanics of a viral capsid lattice and protein-based nanocoating, including flexibility, mechanical strength and remarkable self-repair capacity after mechanical damage; (iii) proof of principle that chemical additives can modify the dynamics and mechanics of a viral capsid lattice or protein-based nanocoating, and improve their applied potential by increasing their mechanical strength and elasticity. We discuss the implications for the development of mechanically resistant and compliant biocoatings precisely organized at the nanoscale, and of novel antiviral agents acting on fundamental physical properties of viruses.
\end{abstract}

Received 17th June 2015 Accepted 30th July 2015

DOI: $10.1039 / c 5 n r 04023 j$

www.rsc.org/nanoscale tested as new vaccines; nanocarriers and nanocontainers for targeted drug delivery; contrast agents for medical imaging; nanobiosensors; nanoreactors; light harvesting devices; and templates to produce monodisperse inorganic or metallic NPs for building improved catalysts, sensors, batteries, electronic circuits and memory devices. ${ }^{2-8}$

In contrast to the diversity of spheroidal ("three-dimensional") or filament-like ("mono-dimensional") protein assemblies, few types of planar ("bi-dimensional", 2-D) protein lattices are known in Nature. ${ }^{9}$ Examples are S-layers, the outermost component of cell walls in archea and many bacteria, ${ }^{10,11}$ and hydrophobin coatings in many fungi. ${ }^{12,13}$ Like viral capsids, these planar protein lattices are self-assembling, stable and highly ordered, because the protein units they are made of are able to self-associate. Inspired by these natural protein assemblies, some non-natural 2-D protein lattices have been engineered. ${ }^{14-17}$

Using a different, template-based approach, fibrin sheets were assembled on micro-patterned structured surfaces ${ }^{18}$ and a variety of 2-D arrays of proteins or virus particles have been obtained by attachment to patterned functionalized templates. $^{8,19}$ Despite the potentialities of templated protein arrays, there is strong applied interest in characterizing, engi-
Centro de Biología Molecular "Severo Ochoa" (CSIC-UAM), Universidad Autónoma de Madrid, Cantoblanco, 28049 Madrid, Spain.E-mail: mgarcia@cbm.csic.es $\dagger$ Electronic supplementary information (ESI) available. See DOI: 10.1039/ c5nr04023j 
neering and expanding the toolkit of self-assembling 2-D protein lattices for nanotechnological uses. ${ }^{9-17,19-25}$ Advantages of the latter include easy and inexpensive production, potential for coating non-patterned, unmodified surfaces, and inbuilt functionalizable elements that are presented in tight, highly ordered arrays. 2-D protein lattices and arrays, generally functionalized with other biomolecules, organic compounds or inorganic NPs, are promising materials for multiple uses, including: coating of non-biological implants; specific biotemplating for cell tissue regeneration; masking during wetetching; and components of catalytic, electrical, electronic, optical or other devices whose enhanced or unique features rely on vast numbers of nanoscale-organized functional elements displayed on a very large surface area. ${ }^{8,9,17,19-28}$

Unfortunately, protein assemblies in general still present several issues for many intended applications. One critical issue is that proteins are "soft" materials, and protein-based NPs may be too sensitive to degradation or disruption by chemical agents, temperature, and/or mechanical forces during their production, storage and/or use. ${ }^{29}$ Another issue is that many protein assemblies (e.g., viral capsids) are conformationally dynamic entities. Many of them have a remarkable propensity to undergo induced structural transitions; $;^{30-32}$ in addition, thermal fluctuations at equilibrium make them sample, through large-scale molecular motions, a wide range of conformations around the lowest free-energy state, a process that has been described as "breathing". ${ }^{31,33,34}$ Conformational dynamism of protein assemblies is biologically relevant and may be desirable, or at least indifferent, for some intended uses of NPs and nanomaterials, but it is undesirable for others (e.g., in NPs or nanoarrays that require precise distances between active components). ${ }^{5}$ Thus, the actual use of protein-based NPs and nanomaterials may critically depend on generating the basic knowledge and practical know-how to adequately improve their mechanical and dynamic features.

Viral capsids provide paradigms to study and manipulate the mechanics and dynamics of protein-based NPs and biomaterials at the nanoscale. Structural studies have greatly advanced our knowledge on the physical bases and biological consequences of conformational transitions and equilibrium dynamics in virus particles. ${ }^{30-32}$ In turn, the emerging area of mechanical virology is providing novel insights into properties of virus particles such as elasticity, brittleness and material fatigue, discovering the structural determinants of these properties, and revealing biological adaptations based on mechanical features. ${ }^{35-47}$ Linkages between propensity for structural rearrangements and mechanical elasticity of virus particles are also being uncovered. ${ }^{40,46}$

Here, the capsid protein of human immunodeficiency virus (HIV) has been used as a model to investigate and manipulate the still largely unknown mechanics and equilibrium dynamics of protein-based 2-D materials organized at the nanoscale. In the mature HIV virion, 1000-1500 copies of the capsid protein (CA) form a cone-shaped capsid around a nucleoprotein complex containing the viral genome. ${ }^{48}$ Despite its odd shape, the architecture of the HIV capsid is based, like a

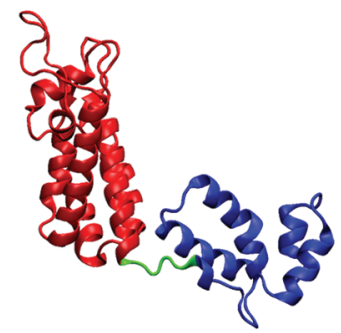

C

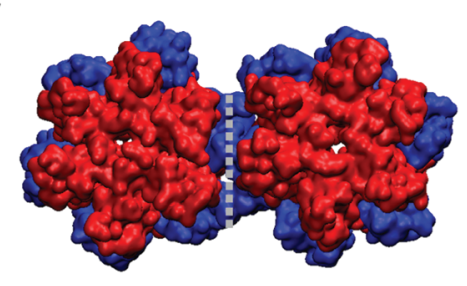

b

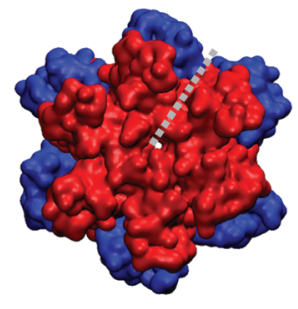

d

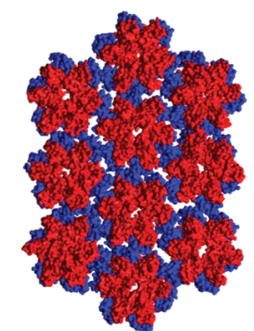

Fig. 1 The mature HIV capsid lattice. The NTD and CTD of CA are respectively colored red or blue. (a) Ribbon diagram of a CA monomer; the linker region is depicted in green. (b) Spacefilling model of a CA hexamer; the dashed line indicates a NTD-NTD hexamerization interface. (c) Two interacting CA hexamers; the dashed line indicates a CTDCTD dimerization interface. (d) A part of the HIV capsid lattice containing 10 hexamers.

some protein layers, on the self-assembly of a hexagonal 2-D protein lattice (Fig. 1) ${ }^{48-51} \mathrm{CA}$ contains two domains (N-terminal domain, NTD, and C-terminal domain, CTD) connected by a flexible linker (Fig. 1a). The hexagonal lattice is made of CA hexamers, in which CA monomers are mainly joined by NTD-NTD and NTD-CTD interactions (Fig. 1b); CA hexamers are bound to neighboring hexamers through CTD-CTD interactions (Fig. 1c and d). Unlike protein layers, the 2-D CA lattice usually folds in the third dimension. In vitro, the CA lattice typically forms hollow open tubes. ${ }^{52-55}$ Within the maturing HIV virion in vivo, the lattice folds into a closed conical shape by insertion of twelve CA pentamers ("defects") at defined positions, as in a fullerene cone..$^{50,51}$

In this study, HIV CA has been used as a building block for the self-assembly of a planar protein lattice (Fig. 1d) that forms a continuous monomolecular nanocoating. This biomaterial provided an excellent model for an unprecedented quantitative analysis using atomic force microscopy (AFM) of the equilibrium dynamics and mechanics of a viral capsid lattice and proteinbased nanocoating. Thermal motions of structural elements and self-repair after mechanical injury were visualized in real time, and chemically induced modifications of its mechanical strength, elasticity and conformational dynamics were quantified.

\section{Results}

Bidimensional self-assembly of the HIV capsid lattice in suspension and on a solid substrate, and structural characterization in a liquid

In vitro, the HIV CA protein self-assembles into long tubes and, occasionally, cone-shaped structures resembling auth- 

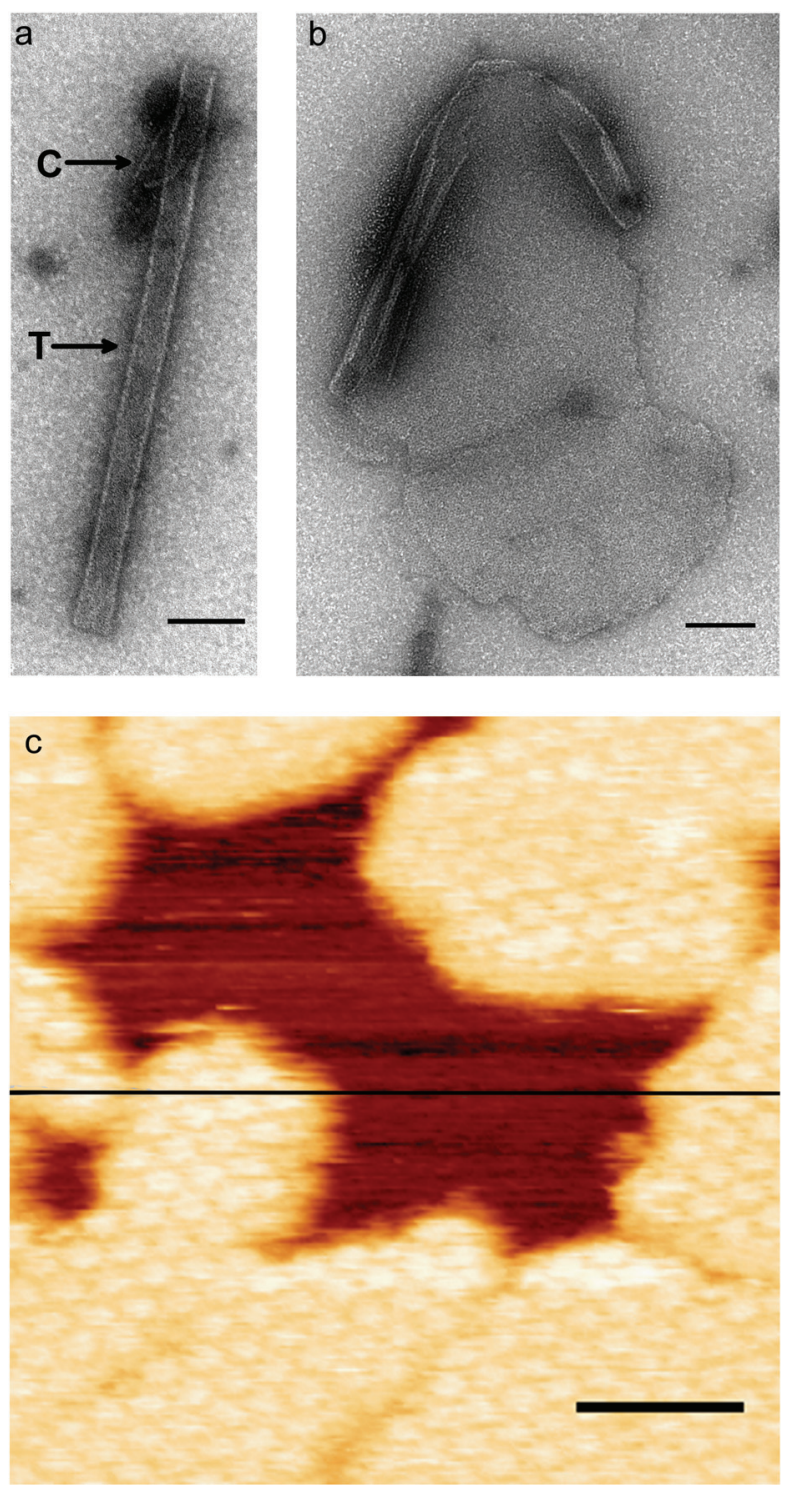

d

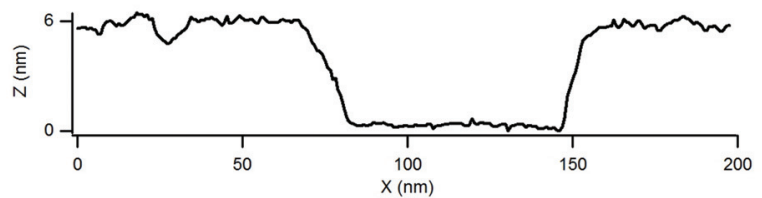

Fig. 2 The self-assembled HIV capsid lattice imaged by transmission EM and AFM. (a) A tube ( $T$, arrow) and a cone-shaped particle ( $C$, arrow, partially covered by the upper end of the tube) and (b) a CA sheet fused to an incomplete tube, all imaged by transmission EM. Scale bars are $100 \mathrm{~nm}$. (c) High-resolution AFM image of the hexagonal CA lattice in a liquid at close to physiological $\mathrm{pH}$, ionic strength and temperature. Brightness of each point in the image is proportional to the height of the sample. Several CA lattice patches surround an uncoated (dark) area. Scale (thick) bar is $40 \mathrm{~nm}$. The thin black line crossing the image corresponds to the height profile shown in panel d. (d) Height profile including the borders of two CA sheets separated by a gap. entic mature HIV capsids ${ }^{52-55}$ (Fig. 2a). However, under the conditions used in the present study, CA formed also abundant, large planar structures (sheets), as revealed by transmission electron microscopy (EM) and AFM imaging of polymerized samples washed with physiological buffer (phosphate-buffered saline, PBS) (Fig. 2b and c). Partially folded tubes fused with sheets were also observed (Fig. 2b), suggesting that CA sheets are built using the same hexameric lattice than authentic HIV capsids, like the tubes and cones observed here and in previous in vitro studies. The assembled material was stable for several months in suspension at $4{ }^{\circ} \mathrm{C}$.

We used solid substrates of a different chemical nature (graphite, glass, silanized glass, mica and polylysine-coated mica) to test their suitability for being coated with preformed CA sheets. The CA sheets attached specifically to a negatively charged surface such as freshly cleaved mica (Fig. 2c). We also tested the possibility of directly assembling the CA lattice onto a solid substrate used as a template. Unpolymerized CA dimers in solution were added to a free mica surface on the AFM stage and incubated, and images were taken (Fig. 3). Efficient polymerization of CA onto the mica surface was achieved in a physiological buffer (PBS); no salt was required. Coverage was dependent on protein concentration, with a nearly continuous CA lattice coating being achieved in less than 30 minutes at a protein (monomer) concentration of $1.3 \mu \mathrm{M}$ under the conditions of the assay (Fig. $3 \mathrm{~d}$ ).

AFM imaging revealed a uniform monomolecular layer of CA (Fig. 2c), with a typical height of $5.5 \pm 0.1 \mathrm{~nm}$ (Fig. 2d) and a topography characterized by the presence of protrusions organized with hexagonal symmetry. Further characterization
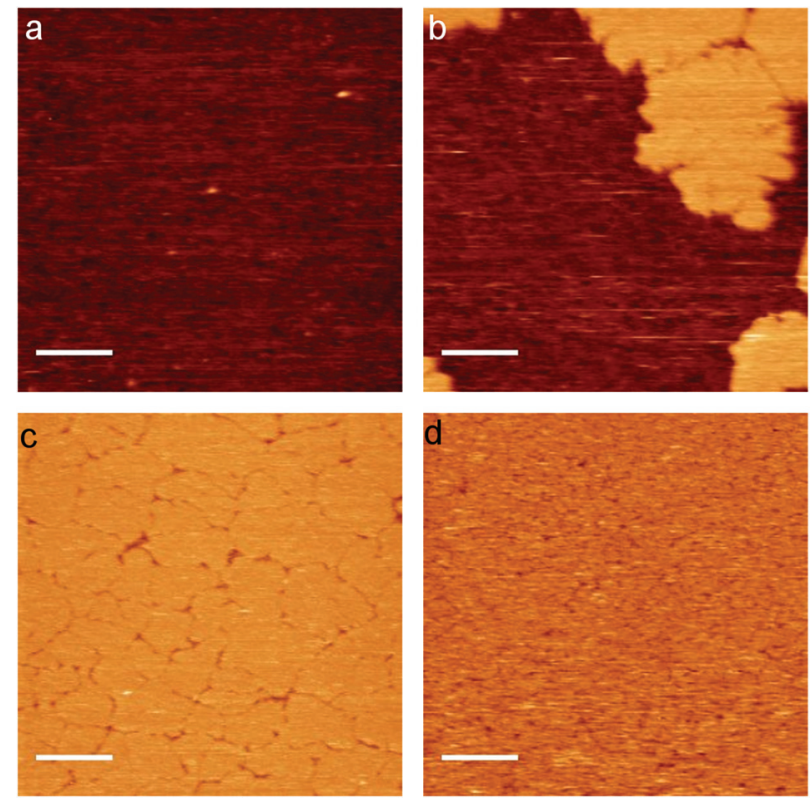

Fig. 3 Direct assembly of the HIV CA lattice onto a mica substrate, imaged by AFM. CA monomer concentration was $0.24 \mu \mathrm{M}$ (a), $0.33 \mu \mathrm{M}$ (b), $0.66 \mu \mathrm{M}$ (c) and $1.32 \mu \mathrm{M}$ (d). Incubation time was $30 \mathrm{~min}$. Scale (white) bar is $400 \mathrm{~nm}$. 
was achieved by imaging at a higher resolution (Fig. 4), exerting very low forces (10-40 $\mathrm{pN}$ ) to avoid possible distortions induced by the cantilever tip. Distortions were also prevented by imaging in jumping mode (as described in Experimental) in which the tip does not contact the sample during the lateral displacements between point measurements. The average distance between the hexamers in the CA lattice in a liquid was independent of the shape and radius of the tip; the value obtained, $9.9 \pm 0.5 \mathrm{~nm}$ (number of measurements, $N=153$ ) (Fig. 4a, upper distribution) was very similar to that found by cryo-EM for vitrified mature HIV capsids. ${ }^{49}$

A comparison between the high-resolution AFM images of the hexameric lattice and the arrangement of hexamers in a

a
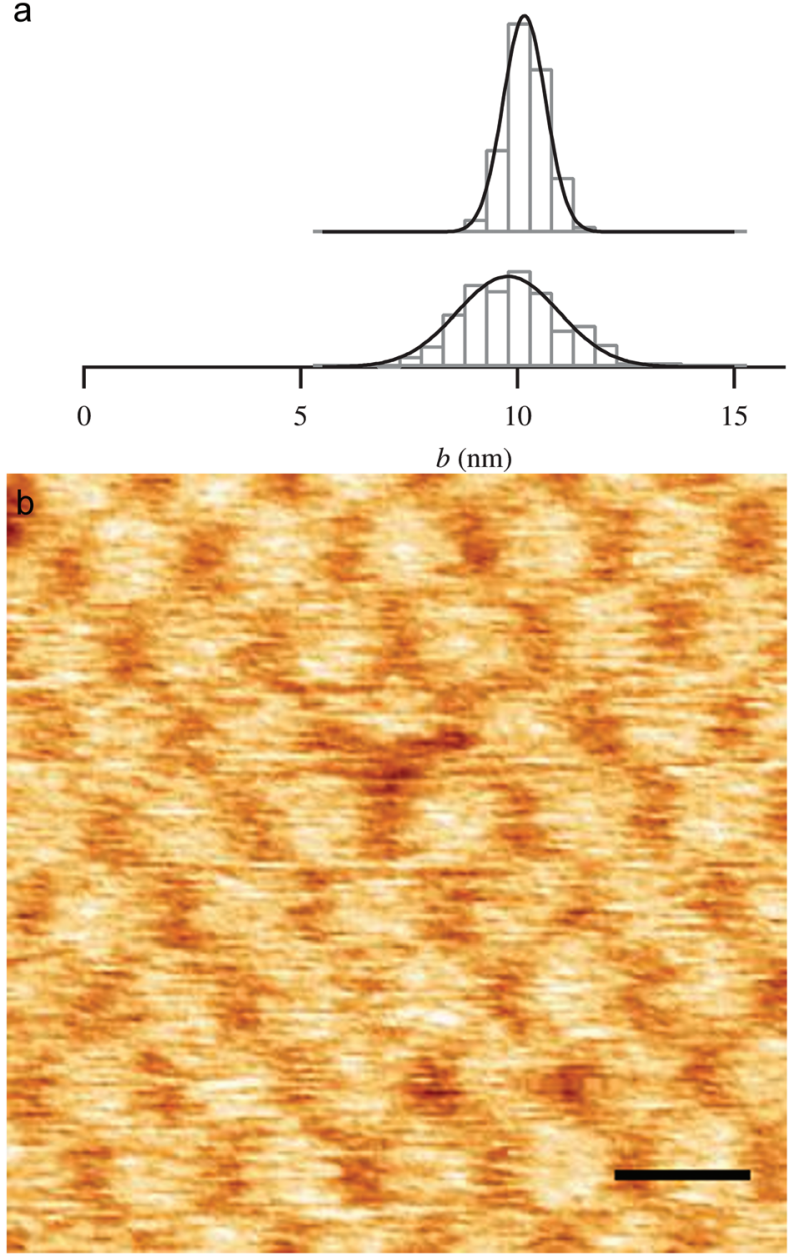

Fig. 4 Structure and dynamics of the HIV CA lattice. (a) Distribution of distances between hexamer centers in the CA lattice assembled in the absence (top) or presence (bottom) of betaine. The respective average values \pm standard deviations (for $N$ measurements) are $9.9 \pm 0.5 \mathrm{~nm}$ $(N=153)$ and $9.7 \pm 1.7 \mathrm{~nm}(N=343)$. These values correspond to the lattice parameter $b$. The widths of the Gaussian fittings for the CA lattice assembled in the absence or presence of betaine were $1.39 \pm 0.02 \mathrm{~nm}$ $(N=153)$ and $3.4 \pm 0.2 \mathrm{~nm}(N=343)$ (see the text and Video $S 1 \dagger)$. (b) High-resolution AFM image of the CA lattice showing a random highamplitude, transient displacement of individual structural elements (dark gap above image center; see the text and Video S1 $\dagger$ ). Scale bar is $10 \mathrm{~nm}$. structural model of the complete mature HIV capsid ${ }^{49-51}$ (Fig. S1 of the ESI $\dagger$ ), allowed us to unambiguously identify the orientation of the protein monomers in the CA sheet. The $\mathrm{N}$-terminal domains NTD (colored red in Fig. S1a and S1b $\dagger$ ) form the protrusions that make up the upper layer, while the C-terminal domains CTD (colored blue in Fig. S1a and S1b †) form the lower layer attached to the substrate. Consistent with this observation, in the CA hexamer upper face, the protruding parts of the NTDs show low charge density with some excess negative charge, while in the lower face the protruding parts of the CTDs show high charge density with excess positive charge (Fig. S2 $\dagger$ ); such charge distribution favors the electrostatic interaction of the CTDs in the lower face of the hexamer with the negatively charged mica surface. This orientation of the CA lattice on the substrate corresponds to that of the capsid in the mature HIV virion, with the NTD layer outside and the CTD layer inside. HIV may have evolved an excess positive charge in its capsid inner wall to neutralize some negative charges of the nucleic acid in the nucleoprotein complex, as found in other viral capsids also. The self-assembly of CA into large sheets (both in suspension and onto a solid substrate) allowed us to use AFM for an unprecedented (to our knowledge) quantitative characterization of the equilibrium dynamics and mechanics of a 2-D protein lattice in a liquid, in close to physiological conditions.

\section{Visualization and quantification by AFM of large-scale equilibrium dynamics (breathing) in the HIV capsid lattice}

AFM not only allowed characterization of the equilibrium parameters of the HIV CA lattice in a liquid, in close to physiological temperature, $\mathrm{pH}$ and ionic strength, but also uniquely allowed a direct quantification of its equilibrium dynamics under these conditions, by directly visualizing the deviations from the lowest free energy conformation through "breathing" motions (Video $\mathrm{S} 1 \dagger)$. Large-scale $(>1 \mathrm{~nm})$ motions in proteins and protein assemblies may take up to many seconds. ${ }^{56}$ Scanning of each hexamer in the CA lattice with our AFM system took about $10 \mathrm{~s}$ for the smaller images, but still yielded quite sharp images of individual hexamers (Fig. 4b and Video S1 $\dagger$ ). These observations indicated that each AFM image represents a fairly sharp snapshot of the actual relative positions of the structural elements in the lattice, and that temporal resolution was enough to detect in real time slow, large-scale relative motions. As each hexamer is indistinguishable from each other, it was very difficult to follow changes in the position of individual hexamers over long times (Video S1†). Thus, we applied the ergodic principle (which implies that the space average is equivalent to the time average) to calculate the breathing amplitude by determining the distribution of distances between the centers of many neighboring hexamers (Fig. 4a), as described in Experimental. Under the near-physiological conditions used with no added ligands, the calculated breathing amplitude was $1.38 \pm 0.02 \mathrm{~nm}(N=153)$ at $25{ }^{\circ} \mathrm{C}$ (Fig. 4a, upper distribution).

Imaging the temporal evolution of the CA lattice also allowed the detection of transient defects in the lattice (Video 
S1† and Fig. 4b). A close visual inspection of these defects indicates that they arise as a consequence of disrupted NTDNTD and/or NTD-CTD interactions in the upper layer that are later re-established, while CTD-CTD interactions are not disrupted. This observation is consistent with the much lower strength of the hexamerization interfaces involving NTDs in each hexamer, relative to the dimerization interfaces involving CTDs of neighboring hexamers. ${ }^{57}$

\section{Mechanical elasticity of the HIV capsid lattice}

In addition to its suitability to visualize viral capsid breathing motions, the HIV CA lattice allowed us to investigate in detail the intrinsic mechanical properties of a protein lattice using AFM. To characterize the elastic properties of CA sheets, we performed indentation experiments to obtain force-distance curves (Fig. 5a) over a range of speed ( 60 to $\left.6000 \mathrm{~nm} \mathrm{~s}^{-1}\right)$. The response was kept under the elastic regime by limiting as much as possible the total indentation (to $<2 \mathrm{~nm}$, less than one-third of the lattice thickness) (Fig. 5a). The spring constant of the
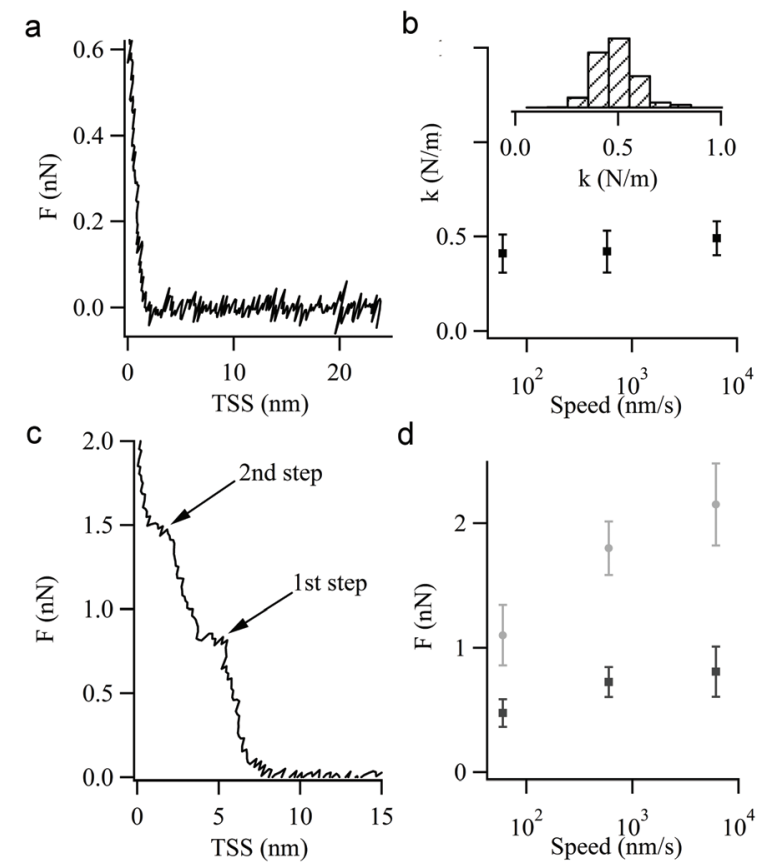

d

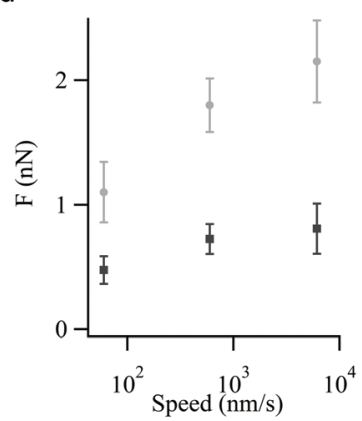

Fig. 5 Mechanical analyses of the HIV CA lattice. (a) A representative force versus tip-sample separation (TSS) curve obtained by indenting the CA lattice under the elastic regime, using low force and shallow $(<2 \mathrm{~nm})$ indentations. Many curves like this one were used to calculate the average spring constant $k_{\mathrm{s}}$. (b) Independence of $k_{\mathrm{s}}$ with the loading rate. Average values \pm standard deviations are indicated. The inset shows the overall distribution of $k_{\mathrm{s}}$ values (average $k_{\mathrm{s}}=0.44 \pm 0.11 \mathrm{~N} \mathrm{~m}^{-1} ; N=$ 79). (c) Representative force versus TSS curve obtained by indenting the CA lattice out of the elastic regime, showing disruption of the lattice in two steps. For a quantitative analysis of these disruption events see the text, Fig. S3 $\uparrow$ and Table 1. (d) Dependence of the rupture force with the loading rate for the first (black squares) or second (grey circles) disruption step. Average values \pm standard deviations are indicated. 32, 19 and 12 experiments were carried out at rates of 60,600 and $6000 \mathrm{~nm} \mathrm{~s}^{-1}$, respectively. lattice, $k_{\mathrm{s}}$, was calculated as described in Experimental. Elasticity did not vary significantly within the explored range of loading rates (Fig. 5b), and the average $k_{\mathrm{s}}$ value obtained was $0.44 \pm 0.11 \mathrm{~N} \mathrm{~m}^{-1}(N=79)$ (inset in Fig. $5 \mathrm{~b}$ ).

\section{Mechanical strength of the HIV capsid lattice and disruption pathway}

We next determined the mechanical (compressive) strength of the CA sheets by dynamic force spectroscopy, to obtain rupture forces at different loading rates (pushing speeds $\sim 60$ to $6000 \mathrm{~nm} \mathrm{~s}^{-1}$ ). Large sheets were indented far from the edge, to evaluate disruption in the virtual absence of border effects. When CA sheets were brought out of the elastic regime by application of enough force, several force steps (evident changes in the slope of the force-distance curve, likely corresponding to partial disruption events) were observed (Fig. 5c). The number of force steps varied in different experiments, meaning that the mechanical disruption of the lattice does not follow a unique pathway. The disruption pathways were classified depending on the number of force steps observed. Four different pathways (named A, B, C and D) were identified at every explored loading rate (Table 1 ). Pathway $\mathrm{B}$, characterized by the presence of two force steps before final disruption of the lattice (Fig. 5c), was the preferential rupture pathway (58\% of cases). Unless specified otherwise, the following descriptions apply to the dominant pathway B.

Each force step was analyzed separately. The rupture force in each step was dependent on the loading rate, but the increase in rupture force was not proportional to the logarithm of the loading rate (Fig. 5d), implying that the disruption event does not occur as a two-state transition. ${ }^{58}$ The average rupture forces for the first and second disruption events were, respectively, $0.48 \pm 0.11 \mathrm{nN}$ and $1.1 \pm 0.2 \mathrm{nN}$ at $60 \mathrm{~nm} \mathrm{~s}^{-1}$, reaching values of $0.8 \mathrm{nN}$ and $2 \mathrm{nN}$ at $6000 \mathrm{~nm} \mathrm{~s}^{-1}$ (Fig. 5d). For any force step (rupture event), the height between the point where the AFM tip encounters initial resistance, and the end point where further resistance is met, corresponds to the height of the part of the protein layer that fails (loses integrity) or is displaced by the applied force (Fig. S3a $\dagger$ ). The corresponding average values for the first and second disruption events were, respectively, $3.0 \pm 0.5 \mathrm{~nm}$ and $3.5 \pm 0.5 \mathrm{~nm}(N=65)$ (Fig. S3b $\dagger$ ). As justified in the Discussion, the ensemble of results suggests that mechanical breakage of the HIV capsid lattice predominantly occurred in two steps: a first, lower force step in which the upper layer of bound NTD domains is embedded between CTD domains in the lower layer, disrupting at least the NTDCTD interfaces; and a second, higher force step in which the lower layer is disrupted or squeezed. After the two disruption events, a hole with a depth of about $6 \mathrm{~nm}$, that involves the entire protein layer, was formed.

\section{Dynamics of the HIV capsid lattice after mechanical disruption}

In addition to elasticity and mechanical strength, the way any nanomaterial behaves after mechanical injury is critically important for evaluating its suitability for different applications. In the case of biological assemblies, it could also be critical for survival 
Table 1 Mechanical breaking pathways of the HIV CA lattice

\begin{tabular}{|c|c|c|c|c|c|c|c|c|c|}
\hline \multicolumn{6}{|c|}{ CA } & \multicolumn{4}{|c|}{ CA+betaine } \\
\hline \multirow[t]{3}{*}{$h(\mathrm{~nm})$} & $1 \mathrm{st}$ & $6.4 \pm 0.5$ & $6.5 \pm 0.6$ & $6.4 \pm 0.4$ & $6.5 \pm 0.7$ & $6.4 \pm 0.7$ & $6.5 \pm 0.7$ & $6.6 \pm 0.7$ & $6.3 \pm 0.8$ \\
\hline & 2nd & & $3.1 \pm 0.7$ & $3.5 \pm 1.2$ & $4.1 \pm 0.8$ & & $3.5 \pm 0.9$ & $3.9 \pm 1.0$ & $4.4 \pm 0.5$ \\
\hline & $3 \mathrm{rd}$ & & & $1.7 \pm 0.7$ & $2.6 \pm 0.7$ & & & $1.8 \pm 0.9$ & $2.7 \pm 0.7$ \\
\hline & 2nd & & $1.15 \pm 0.25$ & $0.78 \pm 0.22$ & $0.64 \pm 0.16$ & & $1.8 \pm 0.3$ & $1.2 \pm 0.3$ & $0.83 \pm 0.29$ \\
\hline & $3 \mathrm{rd}$ & & & $1.25 \pm 0.20$ & $0.98 \pm 0.25$ & & & $2.0 \pm 0.2$ & $1.27 \pm 0.25$ \\
\hline & 4th & & & & $1.40 \pm 0.22$ & & & & $2.0 \pm 0.2$ \\
\hline
\end{tabular}

${ }^{a}$ For each breaking pathway (A, B, C or D), relative probability of occurrence (\%), jump height $(h)$ and force $(F)$ for each disruption step (1st, 2nd, $3 \mathrm{rd}, 4$ th) in the absence (CA) or presence of betaine (CA/betaine) are indicated. Significantly different values ( $t$-test with $p$-value 0.05$)$ are highlighted in boldface.

of the organism. We have uniquely analyzed how a viral capsid lattice responds to local mechanical injury.

We investigated different scenarios, depending on the position of the disrupted area (Fig. 6 and Video $52 \dagger$ ). If the indentation point was far from the border of a sheet, the hole formed was fully repaired in a "self-healing" process (Fig. 6a). This self-repair occurred in every case analyzed, and progressed until no scar in the lattice could be detected, usually after some minutes. Analyses of single force traces when a hole was observed showed a maximal indentation of $6.2 \pm$ $1.1 \mathrm{~nm}(N=24)$ (Fig. S3a $\dagger$ ), corresponding approximately to the thickness of the protein lattice. In contrast, in most of the images taken shortly after opening a hole, the hole no longer went through the entire layer, as its depth had been reduced on average to $2.9 \pm 1.1 \mathrm{~nm}(N=24)$ (Fig. S3c and S3d $\dagger)$. Thus, it appears that this self-repair process, like the dominant disruption process, occurs in two steps: a very fast one that seals the lower $\sim 3.3 \mathrm{~nm}$ of the CA layer, and a slower one in which the upper $\sim 2.9 \mathrm{~nm}$ of the layer is also sealed. The first and second self-healing steps could thus respectively involve the reassociation of CTDs and NTDs in the lattice, and correspond to the reverse of the second and first disruption steps (see the model in Discussion).

If the indentation point was close to the edge of the sheet, a crack opened from this point to the edge, and this fracture evolved in two different ways: some fractures were sealed, leaving a hole that was later repaired as described in the above paragraph (Fig. 6b, CA sheet on the upper part of each image). In contrast, other fractures were not sealed, but the lattice evolved by reshaping, and decreasing the perimeter of the patch through an increase in the number of interactions between the lattice elements (Fig. 6b, CA sheet on the lower part of each image).

Modulation by a small compound of the equilibrium dynamics and mechanical properties of the HIV capsid lattice

Improving the dynamic behavior and mechanical properties of "soft" biomaterials may be critical in realizing their suitability
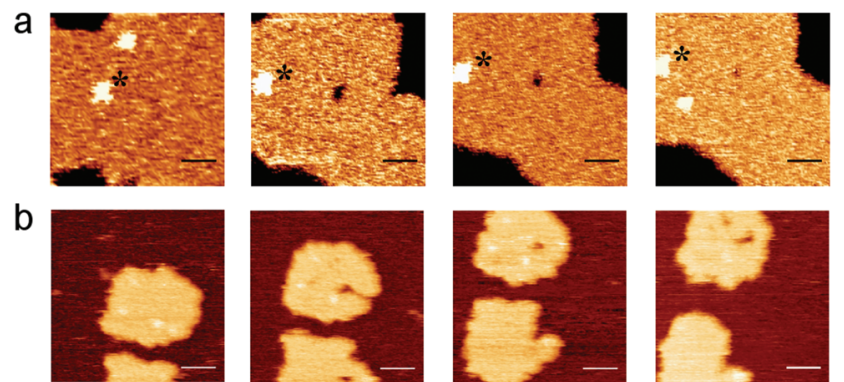

b
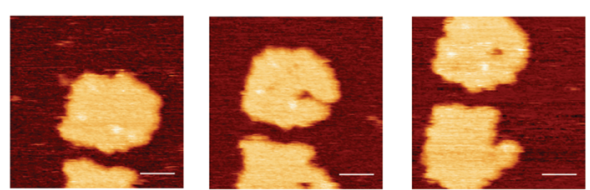

Fig. 6 Disruption and self-repair of the HIV CA lattice. (a) Time course of the disruption and self-healing of a CA sheet indented at a position far from the border. A small contaminant attached to the CA sheet (bright area marked with an asterisk) can be used for orientation. Left image: before indentation. Middle left: after indentation, a hole (black area at the center) is formed. Middle right: the hole shows a reduction in size. Right: the hole has nearly disappeared. (b) Time course of the disruption and self-healing of two small CA sheets indented close to the border. Left image: before indentation. Middle left: after indentation of the CA patch in the upper part of the image, a hole and a fracture that connects the hole with the border were formed. Middle right: the fracture is repaired but the hole remains. Eventually, these holes were also self-repaired (not shown). Between acquisition of the middle left and middle right images, the CA patch at the bottom of each image was also indented. In this case a large, wider fracture occurred. Right image: the fracture on the patch at the bottom widened and caused a restructuring of its shape. All scale bars are $40 \mathrm{~nm}$.

for many nanotechnological applications. In addition, the ability to manipulate those properties allows novel structureproperty-function analyses to provide further insights into virus biology, and guide the design of novel antiviral drugs based on modifying capsid mechanics and/or dynamics. To provide proof-of-principle on the possibility to chemically manipulate the equilibrium dynamics and mechanics of the HIV capsid-like lattice, we have explored the effects of adding the cosolvent betaine. This compound binds proteins in general, and promotes and enhances the assembly of mature HIV capsid-like particles. ${ }^{59}$ 

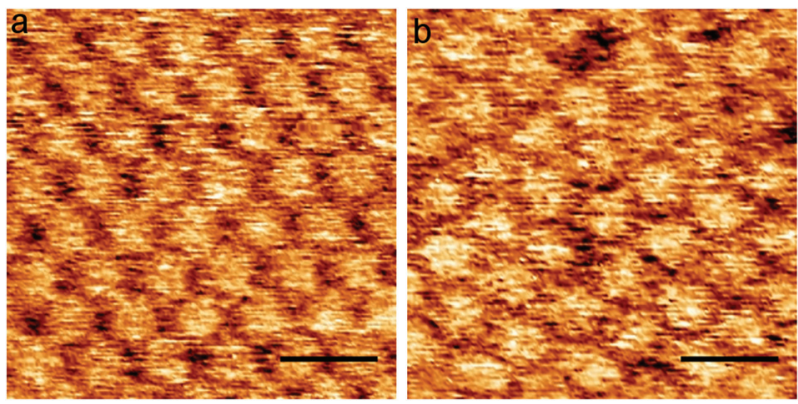

Fig. 7 Topography of the HIV CA lattice and spatial distribution of hexamers in the absence or presence of betaine. High-resolution AFM images taken in the absence (a) or presence (b) of betaine. Scale bar is $15 \mathrm{~nm}$.

Effect of betaine on capsid lattice breathing. The thickness $(5.9 \pm 0.1 \mathrm{~nm})$ and the average distance between the hexamer centres $(9.7 \pm 1.7 \mathrm{~nm})(N=343)$ of the CA sheets assembled and analyzed in the presence of betaine $(0.5 \mathrm{M})$ were indistinguishable from those obtained in its absence in parallel measurements. However, there was a dramatic increase in the dynamic disorder of the structural elements in the lattice (Fig. 7) and the amplitude of the breathing motions, from 1.38 $\pm 0.02 \mathrm{~nm}(N=153)$, in the absence of betaine to $3.4 \pm 0.2 \mathrm{~nm}$ $(N=343)$, in its presence, at $25^{\circ} \mathrm{C}$ (Fig. $\left.4 \mathrm{a}\right)$.

Effect of betaine on capsid lattice mechanics. The mechanical effects of betaine were determined in indentation experiments under the elastic regime at a loading rate of $60 \mathrm{~nm} \mathrm{~s}^{-1}$. Analysis of force-distance curves similar to that shown in Fig. 5a yielded a spring constant of $0.28 \pm 0.15 \mathrm{~N} \mathrm{~m}^{-1}(N=23)$ in the presence of betaine, a value that was significantly lower (by $\sim 30 \%$ ) than that obtained in its absence in parallel experiments. Thus, this compound increased substantially the mechanical elasticity of the CA lattice. Application of higher forces showed that the lattice was disrupted following the same pathways in the presence as in the absence of betaine; pathway B occurred with a similar frequency and was still dominant, although the frequencies of the other disruption pathways changed (Table 1). Importantly, and irrespective of the breaking pathway followed, the mechanical strength of the protein lattice was substantially increased in the presence of betaine. For the dominant breaking pathway $\mathrm{B}$, the rupture force values for the first and second partial disruption events increased by $\sim 28 \%$ and $\sim 59 \%$, respectively (Table 1 ). Thus, binding a small ligand (betaine) to the HIV capsid lattice was enough to substantially increase its large-scale breathing amplitude, mechanical elasticity and resistance to rupture.

\section{Discussion}

The biologically evolved self-assembling capacity of HIV CA allowed its 2-D polymerization, both in suspension and onto an inorganic substrate. In the latter case, efficient self-assembly of CA into a continuous lattice occurred in physiological buffer at ambient temperature; it did not require a high salt concentration, ${ }^{52-54}$ macromolecular crowding agents ${ }^{55}$ or the use of a CA-NC construct that could condense onto a nucleic acid molecule added to the reaction. ${ }^{60}$ The lattice structure and parameters were unchanged relative to those in the authentic HIV capsid. The results demonstrated the possibility of easily achieving full biocoating of a non-biological substrate with a single-molecule-thick protein layer having a highly regular (hexagonal lattice) architecture. The protein domain (NTD) that forms the outermost layer in this coating could be readily functionalized by genetic or chemical engineering for different applications (e.g., tissue regeneration). In the present study, we focused in using this 2-D virus protein lattice as a model to provide novel, quantitative insights into the mechanical properties and equilibrium conformational dynamics of both a viral capsid and a "soft" protein nanocoating, and to explore the possibility of modifying those properties for the design of improved biocoatings and novel antivirals.

\section{Quantifying and manipulating equilibrium dynamics in a virus-based protein lattice}

The techniques available so far to quantify large-scale motions in biological assemblies and nanocoatings are few and technically involved. ${ }^{32,61} \mathrm{X}$-ray crystallography and cryo-EM, as well as cryoelectron crystallography for 2-D lattices, are powerful techniques for determining at high-resolution the equilibrium structure of viruses and other bioassemblies. They may also reveal aspects of their equilibrium dynamics, albeit restrictions due to crystal packing and/or (especially) very low temperatures during data collection must be taken into account. Limited proteolysis-mass spectrometry (MS), hydrogen exchange-MS and nuclear magnetic resonance spectroscopy (NMR) have provided important insights into the equilibrium dynamics of viral particles in solution. ${ }^{31}$ In addition, solid-state NMR spectroscopy analysis recently suggested that a significant proportion of the overall structure in a protein S-layer is disordered. ${ }^{25}$ However, these techniques are complex, may not be applicable to substrate-bound nanocoatings, provide only partial information on dynamics, and give results pertaining to average ensembles of molecules. The present study supports the unique use of AFM imaging as a complementary, singlemolecule approach that uniquely allows direct visualization in real time and quantification of large-scale, slow breathing motions of individual structural elements in viral capsid lattices and ordered protein coatings. These measurements can be carried out under near-physiological conditions and temperature.

The same lattice parameters were obtained by cryo-EM for deep-frozen, vitrified samples of mature HIV capsids, and by AFM for self-assembled, chemically untreated CA sheets in a liquid at ambient temperature. These parameters must, thus, correspond to the actual equilibrium positions of the structural elements (NTDs and CTDs) in the HIV CA protein lattice. In addition, AFM imaging allowed us to: (i) visualize the motions of these structural elements; (ii) determine the breathing amplitude of the HIV CA lattice $(\sim 1.4 \mathrm{~nm})$; (iii) pre- 
cisely quantify variations in breathing amplitude (a 250\% increase) upon addition of a model effector molecule, betaine; (iv) individually detect transient openings in the lattice; and (v) propose a simple model of equilibrium dynamics of the HIV capsid lattice in a liquid. In this model, the stronger CTDCTD interactions at the interhexamer interfaces contribute to keep the capsid lattice lower layer (made of CTDs) relatively static. In contrast, the thermally-induced motions in the flexible CTD-NTD linker peptide in each CA subunit (Fig. 8a), and the transient disruption of the very weak NTD-NTD and NTDCTD interactions at the intrahexamer interfaces, are likely responsible for large breathing motions in the capsid upper layer (made of NTDs). Betaine has some preference for binding hydrophobic residues like Ala, Pro and Phe in proteins. ${ }^{62}$ We tentatively suggest that betaine could disrupt some intersubunit interactions within or between CA hexamers by preferentially binding Pro32, Ala42 at NTD-NTD interfaces, Ala64 and Ala65 at NTD-CTD interfaces in each hexamer, and/ or Ala204 at the small CTD-CTD interfaces close to the threefold axes in the lattice ${ }^{50,51}$ thus increasing breathing motions.

The results did reveal the amplitude of breathing motions of the CA lattice as a nanocoating on a solid surface, but one

a
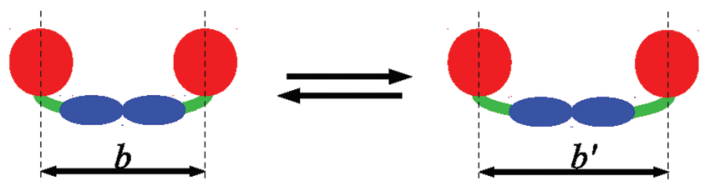

b
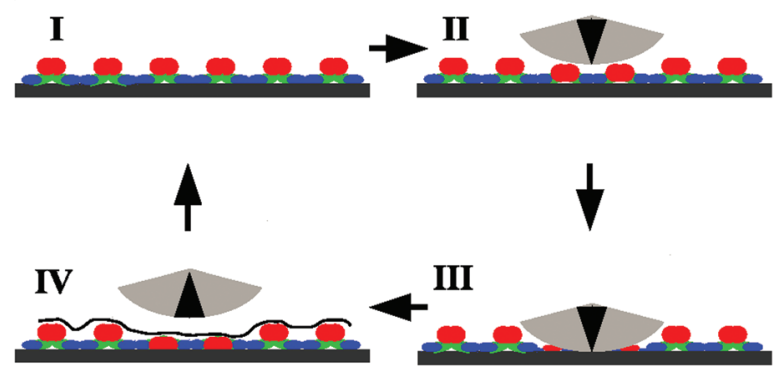

Fig. 8 Simplified models of HIV CA lattice dynamics, mechanical disruption and self-repair. NTDs, CTDs and linker regions are respectively colored red, blue or green. (a) Extension and contraction of the linker region, together with (not shown) disruption and restoration of NTDNTD and/or NTD-CTD interfaces, would allow large-scale, slow "breathing" motions around the equilibrium position altering the lattice parameter (distance between hexamer centres) in the HIV capsid from b to $b^{\prime}$ and back to $b$. (b) Two-step mechanical disruption and self-repair of the CA lattice. I: Non-perturbed state. II: Indentation with the AFM tip (gray sector) forces NTDs below the tip to be encased in the space between CTDs, which is widened; this event would correspond to the first jump observed in the force trace (Fig. 5c). III: Further indentation causes a collapse of the domains and/or their lateral displacement; this event would correspond to the second jump observed in the force trace (Fig. 5c). IV: Immediately after the cantilever tip is retracted, NTDs are reformed and/or displaced back to the gap between CTDs, the hole is closed but a depression remains. Back to I: after some time, the NTDs are lifted to their initial positions above the CTD layer, and the selfhealing process is completed. could ask whether this amplitude would be similar in the unsupported CA lattice or authentic HIV capsid. The available evidence suggests that this may be the case: (i) the observed breathing motions affect essentially the outer NTD layer which is not connected to the substrate, and is only weakly bound to the inner CTD layer by a flexible linker and low-affinity interactions; thus, the same breathing amplitude would be expected for the NTDs in the capsid, either inside the virion or released into the cytoplasm upon infection. (ii) The CTD-CTD interfaces are energetically stronger than the NTD-NTD and NTD-CTD interfaces; thus, it could be expected that the inner CTD layer may contribute little to the global breathing of the mature HIV capsid. (iii) The fast migration of lattice elements during self-repair of fractured CA sheets indicates that the inner CTD layer is only loosely bound to the substrate by transient interactions, being relatively free to move; the CA sheet appears to be attached to the substrate through an energetic potential that depends only on the direction normal to the surface, being free to move in the horizontal plane. (iv) The mature HIV capsid is full of protein and nucleic acid material; thus, similar transient electrostatic interactions may occur between its positively charged inner wall and either the nucleoprotein in vivo or the negatively charged mica substrate in vitro.

\section{Quantifying and manipulating the mechanical behavior of a virus-based protein lattice}

The experimental value obtained for the elastic constant of the actual 2-D lattice was in close agreement with that derived from molecular dynamics simulations on a virtual coarsegrained 2-D lattice of the CA protein. ${ }^{63}$ This agreement supports the validity of this simplified theoretical approach to predict the mechanical elasticity of protein lattices.

We also found that by adding betaine, the elasticity of the CA protein lattice can be increased (by $30 \%$ ) in parallel with a drastic $(250 \%)$ increase in breathing amplitude. It may be tempting to suggest that mechanical elasticity and equilibrium dynamics are linked, with moderate increases in elasticity involving large increases in breathing motions. Indeed, an inextricable linkage has been repeatedly found between mechanical elasticity in a given region of a viral capsid, and propensity for a conformational transition in which that region is involved. ${ }^{40,46}$ Also, a very recent study with a protein S-layer showed a concomitant increase with temperature of both elasticity and structural disorder. ${ }^{25}$ Increasing the elasticity of a nanocoating may have beneficial effects for applications where close contact with a matrix substrate of curved, complex shape is required. However, one should consider that achieving a higher elasticity may also result in substantial increases in equilibrium dynamics, which may or may not be desirable for the intended application.

Small transient gaps were opened and closed in the HIV capsid lattice at equilibrium, as an extreme manifestation of slow breathing motions that are able to transiently disrupt domain-domain interactions in the lattice upper layer. In addition, application of enough mechanical force opened rela- 
tively large holes and cracks through the CA sheets. In most cases the disruption events required forces on the order of $1 \mathrm{nN}$, similar to those needed to disrupt different spherical virus protein particles, as well as the influenza virus lipid envelope. ${ }^{64}$ As with elasticity, the mechanical (compressive) strength of the CA protein sheets was substantially improved by betaine (by $\sim 30 \%$ and $\sim 60 \%$ for the two steps in the dominant disruption pathway). In summary, those results provide proof-of-principle that a chemical additive can make a viral capsid and a protein lattice more elastic and dynamic, and also less brittle, superficially resembling the effects of rejuvenation treatments on some rubber polymer materials.

This HIV capsid lattice showed, in addition, a most remarkable mechanical resilience by being capable of efficient, complete self-repair after mechanical disruption events involving holes or cracks in the material. Self-healing has also been observed in the lipid envelope of influenza virus, ${ }^{64}$ and in cellular protein vaults. ${ }^{65}$ From the experimental data (Fig. 5c and $\mathrm{S} 3 \dagger$ ) and sizes of each CA domain (CTD $2.5 \mathrm{~nm}$; NTD $4.4 \mathrm{~nm}$ ) we propose a model for the rupture and self-repair of the HIV capsid lattice. This model, described in detail in Fig. 8b, proposes a sequential, reversible two-step disruption (and later recovery) of intrahexamer and interhexamer interactions.

\section{Dynamics, mechanics and HIV biology}

From the viewpoint of virology, this study encourages the possibility of designing novel antiviral drugs acting on the mechanics and/or dynamics of a viral capsid. The relevance of these physical features for viral infection has been established for different viruses. ${ }^{30-34,37,38,40,43,46}$ Chemical agents or genetic modifications of the conformational dynamics and/or mechanical elasticity of virus particles have been shown to dramatically impair, for example, the infectivity of rhinovirus ${ }^{33}$ or the infectivity or thermal resistance of the minute virus of mice. ${ }^{40,46}$ Also, truncation of the lipid envelope glycoprotein increased the stiffness of the HIV virion and led to impaired cell penetration and infectivity. ${ }^{43}$

The biological implications of the dynamics and mechanics of the mature HIV capsid lattice revealed here remains to be established, but it is tempting to make a few guesses on their possible influence on capsid assembly and viral genome uncoating. Within the maturing virion the nucleocapsid complex, like the mica surface in our experiments, could act as a template for shaping the capsid, and contribute to define its truncated-cone shape by facilitating the introduction of pentamer "defects" at appropriate positions. The high dynamism and mechanical elasticity of CA could have evolved to facilitate this type of directed capsid closure. Consistent with this possibility, adding betaine in vitro both increased the dynamics and mechanical elasticity of the CA lattice (this study), and enhanced the in vitro assembly of cone-shaped structures similar to authentic mature HIV capsids. ${ }^{59}$

In the face of such a high dynamism and the occurrence of transient defects, the remarkable self-repairing ability of the CA lattice could prevent, once the mature HIV capsid has been released into the infected cell, the untimely exit of some of the capsid contents (which includes not only the nucleocapsid but also other viral and cellular biomolecules). Also, some cellular factors acting during HIV capsid disassembly, like betaine, could facilitate uncoating by modifying the dynamic behavior of the capsid or the rate of self-repair. If the fundamental physical properties of the capsid have a survival value for HIV, as shown for other viruses, ${ }^{40,46}$ compounds that modify the dynamics and/or mechanics of the HIV capsid could be developed into novel anti-HIV drugs.

\section{Conclusions}

This study showed that time-dependent, high-resolution AFM imaging and force measurements can be uniquely combined to characterize, quantify and modify the equilibrium dynamics and mechanics of protein-based 2-D assemblies. By applying this procedure to a viral capsid lattice that was induced to selfassemble as an organized nanocoating, its dynamic disorder at the nanoscale was evaluated by visualizing and determining the amplitude of thermal motions of the lattice structural elements. This analysis also revealed that a viral capsid lattice and protein nanocoating are efficiently self-repaired after mechanical injury. In addition it demonstrated that, at the expense of increasing dynamic disorder, a chemical additive can substantially enhance the mechanical strength and elasticity of a protein lattice. The results (i) open new perspectives to investigate relationships between protein and viral capsid dynamics, mechanics and biology; (ii) provide proof-of principle on the feasibility to improve the mechanical robustness and resilience, and modulate the equilibrium dynamics of protein-based nanocoatings, to increase their suitability for nanotechnological and biomedical applications; and (iii) may lead to the design of novel antiviral drugs based on the modification of biologically relevant physical properties of viral capsids.

\section{Experimental}

\section{Protein expression and purification}

Expression and purification of the HIV-1 CA protein was carried out as previously described. ${ }^{66}$ The protein was essentially pure as determined by analytical size-exclusion chromatography and polyacrylamide gel electrophoresis. After purification, the protein was thoroughly dialysed against sodium phosphate buffer $50 \mathrm{mM} \mathrm{pH} 8$, and stored at $-80^{\circ} \mathrm{C}$ until use.

\section{CA polymerization in vitro}

Assembly of the HIV CA lattice at $25{ }^{\circ} \mathrm{C}$ was triggered by increasing the ionic strength of the solution by adding $\mathrm{NaCl}$ as previously described. ${ }^{55}$ Typical assembly conditions were $50 \mathrm{mM}$ sodium phosphate $\mathrm{pH} 7.8,2.25 \mathrm{M} \mathrm{NaCl}$ and $50 \mu \mathrm{M}$ protein (monomer) concentration. Polymerization of CA was 
monitored by light scattering, measuring the optical density at a wavelength of $350 \mathrm{~nm}$.

\section{Electron microscopy}

EM of the assembled CA structures was performed as previously described. ${ }^{55}$ Briefly, the undiluted CA polymers were deposited onto ionized Formvar/carbon-coated copper grids, fixed for $30 \mathrm{~s}$, negatively stained with $2 \%$ (wt/vol) uranyl acetate (Fluka) for $30 \mathrm{~s}$, dried, and visualized under a JEM-1010 (JEOL) electron microscope.

\section{Adsorption of CA polymers on a substrate and in situ polymerization}

Several substrates with different chemical characteristics were tested for adsorption of pre-assembled CA polymers. The CA sheets were adequately adsorbed onto freshly cleaved mica surfaces. The surface area covered was controlled by dilution of the original sample with $50 \mathrm{mM}$ sodium phosphate, $2.25 \mathrm{M}$ $\mathrm{NaCl}$, typically from $1: 10$ for minimal coverage of the substrate, to $1: 2.5$ for nearly full coverage. Adsorption times varied between $30 \mathrm{~s}$ and 5 min depending on the desired coverage. Low-density CA coverage was used for elasticity measurements, because the large areas without a sample were needed for measuring cantilever sensitivity before and after indentation of the CA lattice. High-density CA coverage was used to analyze consistent dynamic and mechanical properties of a full coating. After the polymer was adsorbed onto the surface, the ensemble was washed several times with PBS and left in the same buffer, in order to perform the AFM experiments in close to physiological conditions. When appropriate, compounds whose effect on the properties of the CA lattice was to be measured were included in the washing and the final sample buffer. For direct polymerization of CA sheets onto the mica substrate, soluble CA at different concentrations in PBS buffer was directly added to the substrate, and incubated for $30 \mathrm{~min}$ at $\sim 25{ }^{\circ} \mathrm{C}$; the sample was washed with PBS to remove the unbound CA and left in the same buffer for AFM analysis.

\section{AFM imaging, dynamic and mechanical analyses}

To image the CA lattice and characterize its structure, dynamic behavior and mechanical properties, we used an AFM (Nanotec Electronica S.L.) operated in a liquid in Jumping Mode ${ }^{67}$ and controlled by WSxM software. ${ }^{68}$ Most of the experiments were performed with ORC8-PS-W cantilevers (Olympus) with a nominal spring constant of $0.1 \mathrm{~N} \mathrm{~m}^{-1}$. At the beginning of each experiment, the actual spring constant of the cantilever in use was determined as previously described. ${ }^{69}$ In order to discard tip size artefacts when measuring lattice parameters, we used cantilevers with a similar nominal spring constant $\left(0.09 \mathrm{~N} \mathrm{~m}^{-1}\right)$ and a nominal tip radius of $10 \mathrm{~nm}$ (BL-AC40-TS, Biolever Mini, Olympus), half that of the ORC8-PS-W cantilevers. Normal force during imaging with both cantilevers was about 10-40 pN. AFM images were processed with WSxM software.

The height of the CA sheet was calculated from a topography histogram obtained with WSxM, which showed two Gaus- sian distributions of values, corresponding to the bottom and the top of the sheet. Then, the height was determined as the distance between the maximum of each distribution.

The breathing amplitude was determined by using ImageJ software ${ }^{70}$ to calculate centre positions of the hexamers within the lattice at different times during a specified time course. First, areas in the images that encircled different hexamers were selected. Then, for each area a mask was generated and the coordinates of each centre were calculated with the particle analysis plug-in included in ImageJ. The coordinates thus obtained for each hexamer were plotted, and the distances between each hexamer and each of its six neighbours in the lattice were determined. After obtaining the distance distributions we calculated the breathing amplitude as the width of the Gaussian fitting of the distribution.

The elastic response of CA sheets was determined by obtaining force-vs.-distance $(\mathrm{Fz})$ curves within the elastic regime, where forward and backward traces overlapped and the maximal indentation was below $\sim 2 \mathrm{~nm}$. CA sheet stiffness was determined by considering the sample and the cantilever as two ideal springs in series, as previously described. ${ }^{71}$

The rupture force was measured by performing indentations large enough to assure complete breakage of the CA sheet. In this case, the necessary sensitivity to recalibrate the curve from volts to newtons was measured in the upper part of the force trace, when the mica surface and the cantilever tip follow the same displacement.

\section{Calculation of electrostatic potential in a CA hexamer}

We used the crystallographic model of the HIV CA hexamer ${ }^{72}$ (Protein Data Bank (PDB) code 3H4E) without the substituted cysteines and APBS software servers ${ }^{73}$ with standard parameters, except for the $\mathrm{pH}$ (set to 8) and the ionic concentration (set to $2.25 \mathrm{M} \mathrm{NaCl}$ ). We set these conditions in order to predict the electrostatic potential during sample incubation and attachment to the surface. Visualization of the electrostatic potential was performed with Visual Molecular Dynamics $(\mathrm{VMD})^{74}$ with a colour code spanning the range -2 to $+2 \mathrm{kT} \mathrm{e}^{-1}$.

Live subject statement. This study involved work with standard recombinant bacteria (Escherichia coli) to produce the CA protein. All experiments were performed in compliance with the relevant laws and institutional guidelines, including the institutional committee that has approved the experiments.

\section{Acknowledgements}

We gratefully acknowledge P.J.de Pablo and J.Gómez-Herrero for scientific discussions, expert advice and support with AFM analysis, and M.A.Fuertes and A.Rodríguez-Huete for technical assistance. A.V. is a recipient of a post-doctoral contract funded by the Spanish Ministerio de Economía y Competitividad (MINECO). This work was supported by grants BIO201237649 from MINECO and S-2009/MAT/1467 from Comunidad 
de Madrid, and an institutional grant from Fundación Ramón Areces. M.G.M. is an associate member of the Centre for Biocomputation and Physics of Complex Systems, Zaragoza, Spain.

\section{References}

1 Protein Nanotechnology, Methods. Mol. Biol, ed. J. A. Gerrard, Humana Press, New York, 2nd edn, 2013, vol. 996.

2 T. Douglas and M. Young, Science, 2006, 312, 873-875.

3 K. T. Kim, S. A. Meeuwissen, R. J. M. Nolte and J. C. M. van Hest, Nanoscale, 2010, 2, 844-858.

4 I. Yildiz, S. Shukia and N. F. Steinmetz, Curr. Opin. Biotechnol., 2011, 22, 901-908.

5 M. G. Mateu, Protein Eng., Des. Sel., 2011, 24, 53-63.

6 A. M. Bittner, J. M. Alonso, M. L. Górzny and C. Wege, in Structure and physics of viruses, ed. M. G. Mateu, Springer, Dordrecht, The Netherlands, 2013, pp. 667-702.

7 J. Glasgow and D. Tullman-Ercek, Appl. Microbiol. Biotechnol., 2014, 98, 5847-5858.

8 F. Li and Q. Wang, Small, 2014, 10, 230-245.

9 D. Papapostolou and S. Howorka, Mol. BioSyst., 2009, 5, 723-732.

10 U. B. Sleytr, B. Schuster, E.-M. Egelseer and D. Pum, FEMS Microbiol. Rev., 2014, 38, 823-864.

11 R. P. Fagan and N. F. Fairweather, Nat. Rev. Microbiol., 2014, 12, 211-222.

12 H. J. Hektor and K. Scholtmeijer, Curr. Opin. Biotechnol., 2005, 16, 434-439.

13 J. Bayry, V. Aimanianda, J. I. Guijarro, M. Sunde and J. P. Latgé, PLoS Pathog., 2012, 8, e1002700.

14 J. E. Padilla, C. Colovos and T. O. Yeates, Proc. Natl. Acad. Sci. U. S. A., 2001, 98, 2217-2221.

15 P. Ringler and G. E. Schulz, Science, 2003, 302, 106-109.

16 Y. T. Lai, N. P. King and T. O. Yeates, Trends Cell Biol., 2012, 22, 653-661.

17 F. Baneyx and J. F. Matthaei, Curr. Opin. Biotechnol., 2014, 28, 39-45.

18 E. T. ÓBrien III, M. R. Falvo, D. Millard, B. Eastwood, R. M. Taylor II and R. Superfine, Proc. Natl. Acad. Sci. U. S. A., 2008, 105, 19438-19443.

19 S. Zhang, Nat. Biotechnol., 2003, 21, 1171-1178.

20 L. De Stefano, I. Rea, A. Armenante, P. Giardina, M. Giocondo and I. Rendina, Langmuir, 2007, 23, 79207922.

21 S. Howorka, Curr. Opin. Biotechnol., 2011, 22, 485-491.

22 C. Horejs, H. Gollner, D. Pum, U. B. Sleytr, H. Peterlik, A. Jungbauer and R. Tscheliessnig, ACS Nano, 2011, 5, 2288-2297.

23 B. Schuster and U. B. Sleytr, Methods Mol. Biol., 2013, 996, 153-175.

24 M. Rothbauer, S. Küpkü, D. Sticker, U. B. Sleytr and P. Ertl, ACS Nano, 2013, 7, 8020-8030.
25 P. de sa Peixoto, C. Roiland, D. Thomas, V. Briard-Bion, R. Le Guellec, S. Parayre, S.-M. Deutsch, G. Jan and F. Guyomarćh, Langmuir, 2015, 31, 199-208.

26 A. Nag and A. R. Dinner, Biophys. J., 2006, 90, 896-902.

27 S. A. Semerdzhiev, D. R. Dekker, V. Subramanian and M. M. Claessens, ACS Nano, 2014, 8, 5543-5551.

28 E. Olçeroğlu, C.-Y. Hsieh, M. M. Rahman, K. K. S. Lau and M. McCarthy, Langmuir, 2014, 30, 7556-7566.

29 M. J. Buehler and Y. C. Yung, Nat. Mater., 2009, 8, 175-188.

30 J. E. Johnson, Adv. Protein Chem., 2003, 64, 197-218.

31 B. Bothner and J. K. Hilmer, in Structural Virology, ed. M. Agbandje-McKenna and R. McKenna, RSC Publishing, Cambridge, UK, 2011, pp. 41-61.

32 M. G. Mateu, Arch. Biochem. Biophys., 2013, 532, 65-79.

33 J. K. Lewis, B. Bothner, T. J. Smith and G. Siuzdak, Proc. Natl. Acad. Sci. U. S. A., 1998, 95, 6774-6778.

34 B. Bothner, A. Schneemann, D. Marshall, V. Reddy, J. E. Johnson and G. Siuzdak, Nat. Struct. Biol., 1999, 6, 114-116.

35 I. L. Ivanovska, P. J. de Pablo, B. Ibarra, G. Sgalari, F. C. MacKintosh, J. L. Carrascosa, C. F. Schmidt and G. J. Wuite, Proc. Natl. Acad. Sci. U. S. A., 2004, 101, 7600-7605.

36 W. H. Roos and G. J. L. Wuite, Adv. Mater., 2009, 21, 11871192.

37 W. H. Roos, R. Bruinsma and G. J. L. Wuite, Nat. Phys., 2010, 6, 733-743.

38 M. G. Mateu, Virus Res., 2012, 168, 1-22.

39 W. H. Roos, I. Gertsman, E. R. May, C. L. Brooks 3rd, J. E. Johnson and G. J. Wuite, Proc. Natl. Acad. Sci. U. S. A., 2012, 109, 2342-2347.

40 M. Castellanos, R. Pérez, C. Carrasco, M. Hernando-Pérez, J. Gómez-Herrero, P. J. de Pablo and M. G. Mateu, Proc. Natl. Acad. Sci. U. S. A., 2012, 109, 12028-12033.

41 P. J. de Pablo and M. G. Mateu, in Structure and physics of viruses, ed. M. G. Mateu, Springer, Dordrecht, The Netherlands, 2013, pp. 519-551.

42 A. Luque and D. Reguera, in Structure and physics of viruses, ed. M. G. Mateu, Springer, Dordrecht, The Netherlands, 2013, pp. 553-595.

43 H.-B. Pang, L. Hevroni, N. Kol, D. M. Eckert, M. Tsvitov, M. S. Kay and I. Rousso, Retrovirology, 2013, 10, 4.

44 M. Hernando-Pérez, E. Pascual, M. Aznar, A. Ionel, J. R. Castón, A. Luque, J. L. Carrascosa, D. Reguera and P. J. de Pablo, Nanoscale, 2014, 6, 2702-2709.

45 M. Hernando-Pérez, S. Lambert, E. Nakatani-Webster, C. E. Catalano and P. J. de Pablo, Nat. Commun., 2014, 5, 4520.

46 M. Castellanos, P. J. P. Carrillo and M. G. Mateu, Nanoscale, 2015, 19, 5654-5664.

47 M. G. van Rosmalen, W. H. Roos and G. J. Wuite, Methods Mol. Biol., 2015, 1252, 115-137.

48 W. I. Sundquist and H.-G. Kräusslich, Cold Spring Harbor Perspect. Med., 2012, 2, a006924.

49 B. K. Ganser-Pornillos, A. Cheng and M. Yeager, Cell, 2007, 131, 70-79.

50 O. Pornillos, B. K. Ganser-Pornillos and M. Yeager, Nature, 2011, 469, 424-427. 
51 G. Zhao, J. R. Perilla, E. L. Yufenyuy, X. Meng, B. Chen, J. Ning, J. Ahn, A. M. Gronenborn, K. Schulten, C. Aiken and P. Zhang, Nature, 2013, 497, 643-646.

52 L. S. Ehrlich, B. E. Agresta and C. A. Carter, J. Virol., 1992, 66, 4874-4883.

53 S. Li, C. P. Hill, W. I. Sundquist and J. T. Finch, Nature, 2000, 407, 409-413.

54 J. Lanman, J. Sexton, M. Sakalian and P. E. Prevelige Jr., J. Virol., 2002, 76, 6900-6908.

55 M. del Alamo, G. Rivas and M. G. Mateu, J. Virol., 2005, 79, 14271-14281.

56 M. Karplus, J. Phys. Chem. B, 2000, 104, 11-27.

57 M. G. Mateu, FEBS J., 2009, 276, 6098-6109.

58 C. Bustamante, Y. R. Chemla, N. R. Forde and D. Izhaky, Annu. Rev. Biochem., 2004, 73, 705-748.

59 A. Lampel, Y. Bram, M. Levy-Sakin, E. Bacharach and E. Gazit, PLoS One, 2013, 8, e60867.

60 S. Campbell and V. M. Vogt, J. Virol., 1995, 69, 6487-6497.

61 K. Henzler-Wildman and D. Kern, Nature, 2007, 450, 964972.

62 Y. L. Shek and T. V. Chalikian, J. Phys. Chem., 2011, 115, 11481-11489.

63 S. D. Hicks and C. L. Henley, Phys. Rev. E: Stat., Nonlinear, Soft Matter Phys., 2010, 81, 030903.

64 S. Li, F. Eghiaian, C. Sieben, A. Herrmann and I. A. Schaap, Biophys. J., 2011, 100, 637-645.
65 A. Llauró, P. Guerra, N. Irigoyen, J. F. Rodriguez, N. Verdaguer and P.J. de Pablo, Biophys. J., 2014, 106, 687695.

66 M. del Alamo, J. L. Neira and M. G. Mateu, J. Biol. Chem., 2003, 278, 27923-27929.

67 F. Moreno-Herrero, J. Colchero, J. Gómez-Herrero and A. M. Baró, Phys. Rev. E: Stat., Nonlinear, Soft Matter Phys., 2004, 69, 031915.

68 I. Horcas, R. Fernández, J. M. Gómez-Rodríguez, J. Colchero, J. Gómez-Herrero and A. M. Baró, Rev. Sci. Instrum., 2007, 78, 013705.

69 J. E. Sader, J. W. M. Chon and P. Mulvaney, Rev. Sci. Instrum., 1999, 70, 3967-3969.

70 C. A. Schneider, W. S. Rasband and K. W. Eliceiri, Nat. Methods, 2012, 9, 671-675.

71 C. Carrasco, A. Carreira, I. A. Schaap, P. A. Serena, J. Gómez-Herrero, M. G. Mateu and P. J. de Pablo, Proc. Natl. Acad. Sci. U. S. A., 2006, 103, 13706-13711.

72 O. Pornillos, B. K. Ganser-Pornillos, B. N. Kelly, Y. Hua, F. G. Whitby, C. D. Stout, W. I. Sundquist, C. P. Hill and M. Yeager, Cell, 2009, 137, 1282-1292.

73 N. A. Baker, D. Sept, S. Joseph, M. J. Holst and J. A. McCammon, Proc. Natl. Acad. Sci. U. S. A., 2001, 98, 10037-10041.

74 W. Humphrey, A. Dalke and K. Schulten, J. Mol. Graphics, 1996, 14, 33-38. 